\title{
Liquid-phase Dehydration of 1-Phenylethanol to Styrene over an Acidic Resin Catalyst
}

\author{
Nazmul Abedin Khan, Jin-Soo Hwang, ${ }^{\dagger}$ and Sung Hwa Jhung ${ }^{*}$ \\ Department of Chemistry and Green-Nano Materials Research Center, Kyungpook National University, Daegu 702-701, Korea \\ *E-mail: sung@knu.ac.kr \\ †Bio-refinerary Research Center, Korea Research Institute of Chemical Technology, P.O. Box, 107, Yusung, \\ Daejeon 305-600, Korea
}

Received January 27, 2011, Accepted March 3, 2011

\begin{abstract}
Dehydration of 1-phenylethanol to produce styrene has been studied in liquid phase with three solid acid catalysts such as H-ZSM-5, H-Y and Amberlyst-15. Amberlyst- 15 shows the highest conversion and styrene yield, suggesting the applicability of a resin catalyst in the dehydration. The good performance of the Amberlyst-15 may be due to high acid concentration and ready diffusion of reactants and products. A possible reaction scheme (such as the formation of styrene from diphenylethylether) has also been suggested.
\end{abstract}

Key Words : Dehydration, 1-Phenylethanol, Amberlyst-15, Liquid-phase, Styrene

\section{Introduction}

Dehydration of alcohols ${ }^{1}$ has been widely studied and used to produce organic olefins, valuable intermediates because of high activity and functionality. The dehydration has usually been carried out with acid catalysts such as mineral acids and solid acids. Various alcohols including methanol, ${ }^{2-6}$ butanol, ${ }^{7,8}$ hexanol, ${ }^{9}$ and glycerol ${ }^{10}$ have been converted into attractive intermediates by using suitable catalysts.

Styrene (ST) is one of the most widely used raw chemicals for polymers and usually produced with dehydrogenation of ethylbenzene (EB) at high temperature by using iron oxide catalysts in the presence of superheated steam. ${ }^{11,12}$ Even though the direct production of ST from EB is widely used, the process has a drawback of high energy consumption. Around $15 \%$ of ST is produced by dehydration of 1phenylethanol $(\mathrm{PhE})$ which is obtained from a process called $\mathrm{PO} / \mathrm{SM}$ (propylene oxide/styrene monomer) ${ }^{13-16}$ Moreover, there was a process ${ }^{15,16}$ to produce $\mathrm{ST}$ by dehydration of $\mathrm{PhE}$ which was obtained from acetophenone. The acetophenone is usually originated from oxidation of EB.

Dehydration of $\mathrm{PhE}$ has been commercially carried out at around $300{ }^{\circ} \mathrm{C}$ in gas phase in the presence of solid acids like titania and alumina. ${ }^{15,16}$ Recently, liquid-phase dehydration has been studied using various catalysts like zeolites (HZSM- $5,{ }^{13,16}$ mordenite ${ }^{14-16}$ and $\mathrm{H}-\mathrm{Y}^{16}$ ), alumina, ${ }^{17}$ polyoxometallates, ${ }^{16}$ and silica-alumina. ${ }^{16}$ Liquid-phase dehydration is very attractive because energy consumption can be minimized due to low operation temperature. However, there has been little study to develop suitable acid catalysts especially solid acid catalysts. Solid acid catalysts are very promising and important because they are environmentfriendly, recyclable and can be separated easily.

So far, to the best of our knowledge, there has been no report to use a resin catalyst ${ }^{18}$ like Amberlyst-15 (AMB-15) in the dehydration of $\mathrm{PhE}$ even though resins have been used widely in acid catalysis. ${ }^{18-22}$ Moreover, resin catalysts have the advantage of high concentration of acid sites, easy production and low cost. ${ }^{18}$ In this work, the dehydration of $\mathrm{PhE}$ with a resin catalyst (AMB-15) will be discussed. The catalyst shows not only high activity but also high yield for ST. Possible reaction scheme from PhE to ST, an intermediate and a heavy product will also be suggested.

\section{Experimental}

The dehydration of 1-phenylethanol (PhE, 98\%, Aldrich) was carried out in liquid phase using a round bottomed 50 $\mathrm{mL}$ three-neck flask equipped with a small rectification column, and a water cooled condenser attached with a vacuum pump. An oil bath was employed to maintain the reaction temperature of $170{ }^{\circ} \mathrm{C}$. The scheme of the reactor is illustrated in Figure 1.

Three different commercial catalysts $(0.1 \mathrm{~g}$ each) like H-

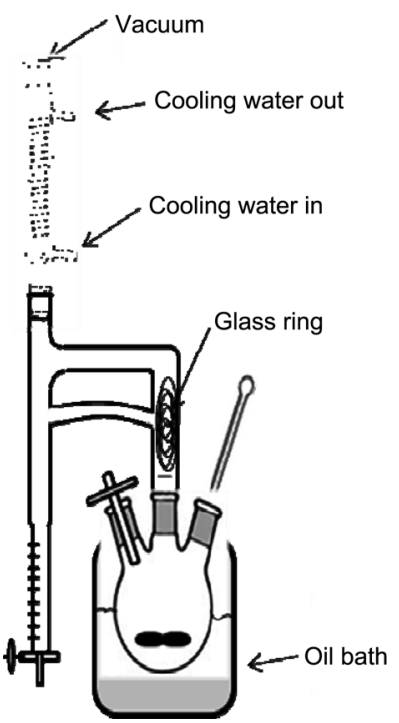

Figure 1. The scheme of the reaction system used in this study. 
Table 1. Textrual properties of the three catalysts

\begin{tabular}{cccc}
\hline Catalysts & $\begin{array}{c}\text { BET surface area, Total pore volume, } \\
\mathrm{m}^{2} / \mathrm{g}\end{array}$ & $\begin{array}{c}\text { Micropore } \\
\text { volume }, \mathrm{cm}^{3} / \mathrm{g}\end{array}$ \\
\hline $\mathrm{H}-\mathrm{ZSM}-5$ & 344 & 0.22 & 0.12 \\
$\mathrm{H}-\mathrm{Y}$ & 486 & 0.35 & 0.20 \\
$\mathrm{AMB}-15$ & 45 & 0.30 & $\sim 0$ \\
\hline
\end{tabular}
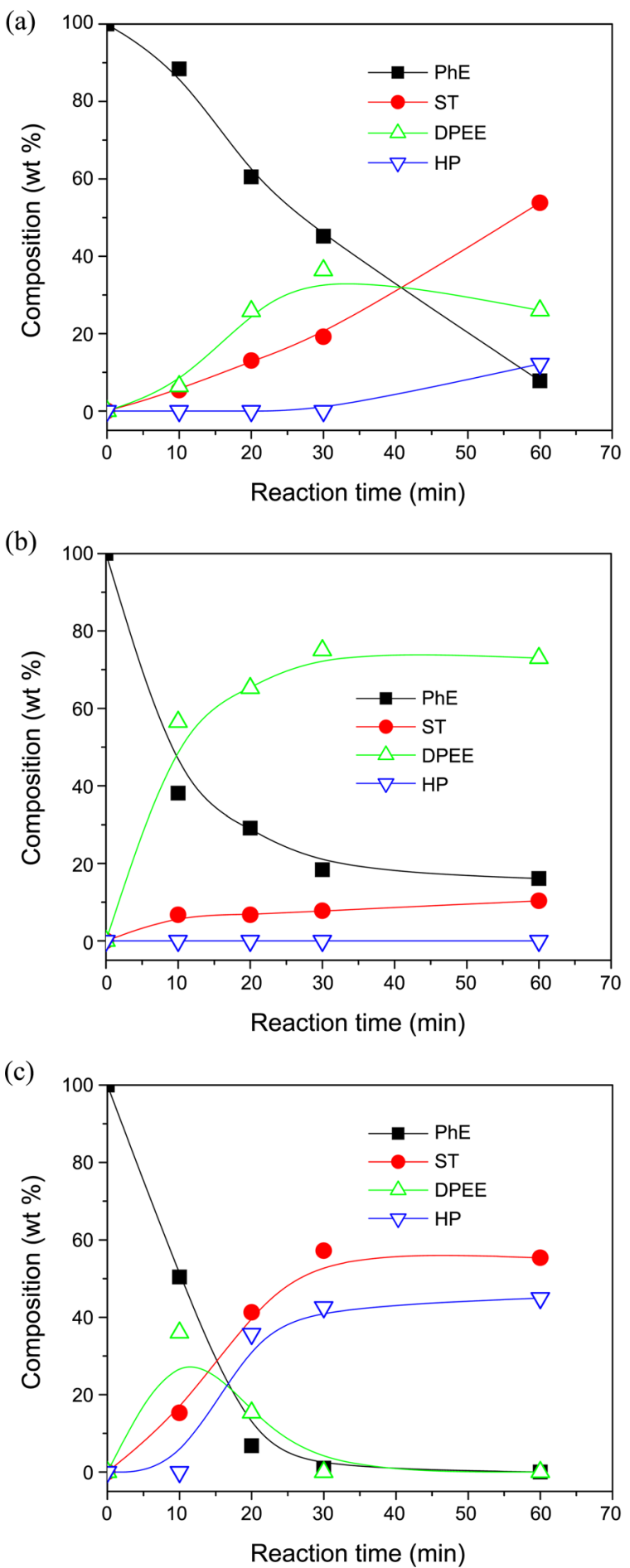

Figure 2. Changes of the compositions of the reaction system depending on the reaction time in the presence of three catalysts: (a) H-ZSM-5; (b) H-Y and (c) AMB-15.
ZSM-5 (Si/Al=23, Zeolyst), H-Y (Si/Al=15, Zeolyst) and Amberlyst-15 (Aldrich, acid capacity is 4.7 mequiv. $\mathrm{H}^{+} / \mathrm{g}$ ) were used in this study. The surface area and pore volumes (Table 1) of the catalysts were obtained from nitrogen adsorption isotherms (shown in Supporting Figure 1). The pore size distributions (shown in Supporting Figure 2) of the catalysts were obtained from the nitrogen adsorptiondesorption isotherms. The nitrogen adsorption-desorption isotherms were obtained using a Micromeritics Tristar II 3020 surface area and porosity analyzer at the temperature of liquid nitrogen $\left(-196^{\circ} \mathrm{C}\right)$ after evacuation at $200^{\circ} \mathrm{C}$ (for $\mathrm{H}-$ ZSM-5 and H-Y) or $110{ }^{\circ} \mathrm{C}$ (for AMB-15).

For solvent-free dehydration, the flask was loaded with 15 $\mathrm{g}$ of 1-phenylethanol and dipped in the hot oil bath for rapid heating to the reaction temperature. The dehydration reaction was also carried out in the presence of 1,2-dichlorobenzene ( $99 \%$, Samchun Chemical) as a solvent. A mixture of $5 \mathrm{~g}$ of PhE and $25 \mathrm{~g}$ of 1,2-dichlorobenzene (o-DCB) was loaded in the flask and $0.1 \mathrm{~g}$ of Amberlyst- 15 was used as the catalyst. The pressure of the reaction system was decreased ( $\sim 0.8 \mathrm{bar}$ ) to remove low boiling portion (water and ST). As the reaction proceeded, distillate fraction containing water and ST was collected in a vessel. After a fixed reaction time, the reaction flask was removed from the oil bath and was allowed to cool down to room temperature. The catalyst was separated from the residual slurry by centrifugation. The styrene rich organic phase and residual slurry were mixed well and analyzed with a FID GC. Products like ST, diphenylethylether (DPEE) and heavy products were identified with authentic samples and GC-MS (Finnigan, MATGCQ).

\section{Results and Discussion}

Comparison of Catalysts. Figure 2 compares the performance (relative composition of reactant and products) of the three catalysts in the $\mathrm{PhE}$ dehydration at $170{ }^{\circ} \mathrm{C}$ depending on the reaction time. The conversion, ST selectivity and ST yield are summarized in Figure 3. As shown in Figures 2 and 3 , the PhE converts quite rapidly over the three catalysts. The performance, especially ST yield, is generally in the order of AMB-15 > H-ZSM-5 > H-Y (Figure 3(c)), suggesting the applicability of a resin catalyst, AMB-15, in the dehydration of $\mathrm{PhE}$ to $\mathrm{ST}$. To compare precisely the relative kinetics of $\mathrm{PhE}$ dehydration, the conversion of $\mathrm{PhE}$ is plotted to follow the first order kinetics (Figure 4). As illustrated in Figure 4 , the rate constants are $k_{\mathrm{AMB}-15}\left(1.6 \times 10^{-1} / \mathrm{min}\right)>$ $k_{\mathrm{H}-\mathrm{Y}}\left(5.4 \times 10^{-2} / \mathrm{min}\right)>k_{\mathrm{H}-\mathrm{ZSM}-5}\left(2.8 \times 10^{-2} / \mathrm{min}\right)$.

Medium-pore zeolites, especially small-sized crystals, have shown good performance in the dehydration of PhE. ${ }^{16} \mathrm{H}-$ ZSM-5 $(\mathrm{Si} / \mathrm{Al}=20)$ has been used in the dehydration to develop a kinetic model. ${ }^{13} \mathrm{H}$-mordenite and $\mathrm{H}-\mathrm{Y}$ have been applied in the dehydration; ${ }^{16}$ however, the yield of ST is poor because large-pore zeolites lead mainly to the diphenylethylether (DPEE) in a wide range of conversion. Similar to a previous work, ${ }^{16}$ ST yield over H-Y is low (Figure 3(c)) because of high selectivity to DPEE even though H-Y shows 

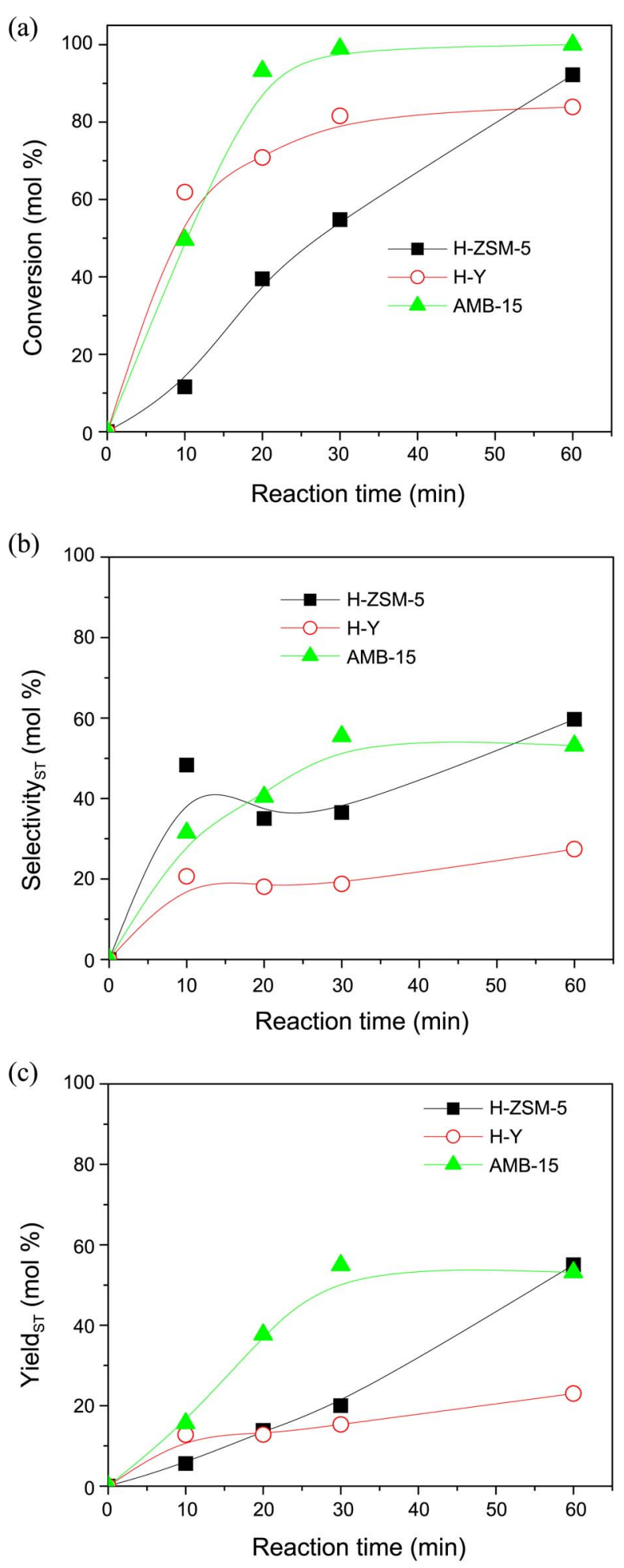

Figure 3. (a) PhE conversion, (b) ST selectivity and (c) ST yield at various reaction times in the presence of three catalysts.

high PhE conversion. H-ZSM-5 has relatively low conversion in a short reaction time even though the ST selectivity is relatively high.

To increase ST selectivity further with the AMB-15, the dehydration of $\mathrm{PhE}$ was carried out in the presence of a solvent, 1,2-dichlorobenzene. As shown in Figure 5, the ST

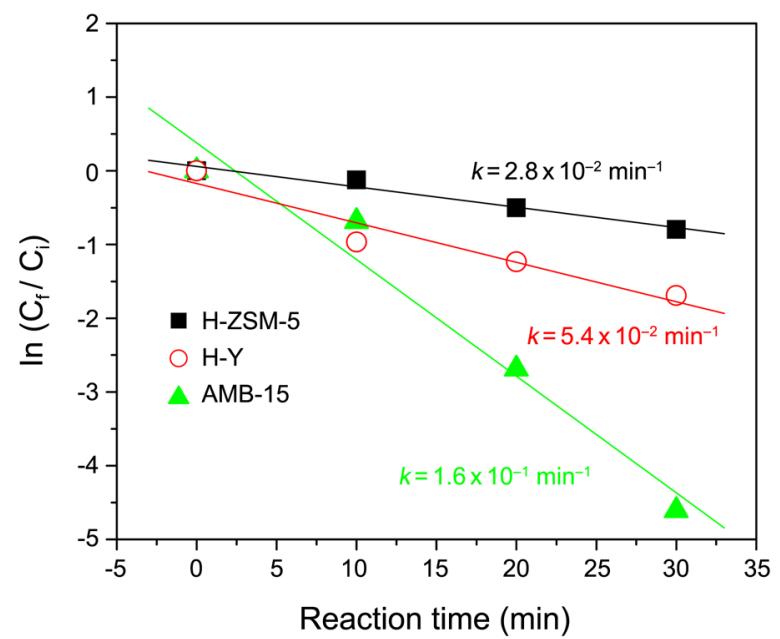

Figure 4. Plots to show the first order kinetics of the $\mathrm{PhE}$ dehydration with the three catalysts. The kinetic constants are also shown.

selectivity can be increased up to $85.5 \%$ at the conversion of $100 \%$ in a selected reaction condition (reaction time: 30 min). The solvent may hinder the formation of heavy products (HP) because the interaction between ST molecules can be hindered with dilution (see below).

Therefore, the AMB-15 catalyst shows the best performance among the three catalysts not only in the conversion but also in the ST yield, suggesting the potential applications of the catalyst in the $\mathrm{PhE}$ dehydration.

Reaction Scheme and Kinetics Over the AMB-15 Catalyst. The mechanism of the dehydration of PhE over the acidic catalysts has been described a few times in open literatures. ${ }^{13-17}$ However, there is a disagreement between research groups. For example, Bertero et al. ${ }^{13,14}$ and Romanova et $a l .{ }^{17}$ have suggested that DPEE can be converted into ST; however, Lange et al. ${ }^{15,16}$ proposed that DPEE can be obtained from ST.

Figure 2 shows the compositions of ST, DPEE and HP, and suggests that ST and DPEE are primary products and HP is a secondary product because primary and secondary products have non-zero and zero initial slopes, respectively. Interestingly, the concentration of DPEE shows a maximum and decreases steadily in the case of H-ZSM-5 and AMB15 , suggesting the DPEE is an intermediate that can be converted into other products like ST or HP. Moreover, it has been agreed on that olefins can be obtained from ether in the MTO (methanol-to-olefin) process. ${ }^{3,4}$ Therefore, the DPEE, produced at the early stage of reaction, may be converted into ST. However, the possibility of DPEE reconversion into $\mathrm{PhE}$ (and finally dehydrated to ST) cannot be ruled out.

The HP concentration increases steadily (Figures 2 and 5) and ST concentration decreases when HP concentration is very high (Figure 5). GC-MS analysis shows that the HP is composed of carbon and hydrogen (chemical formula: $\left(\mathrm{C}_{6} \mathrm{H}_{5}\right)_{4}(\mathrm{CH})_{6}$; FW: 386). Therefore, HP may be mainly obtained from oligomerization of ST. The reaction pathway, in conclusion, may be summarized as Scheme 1. 


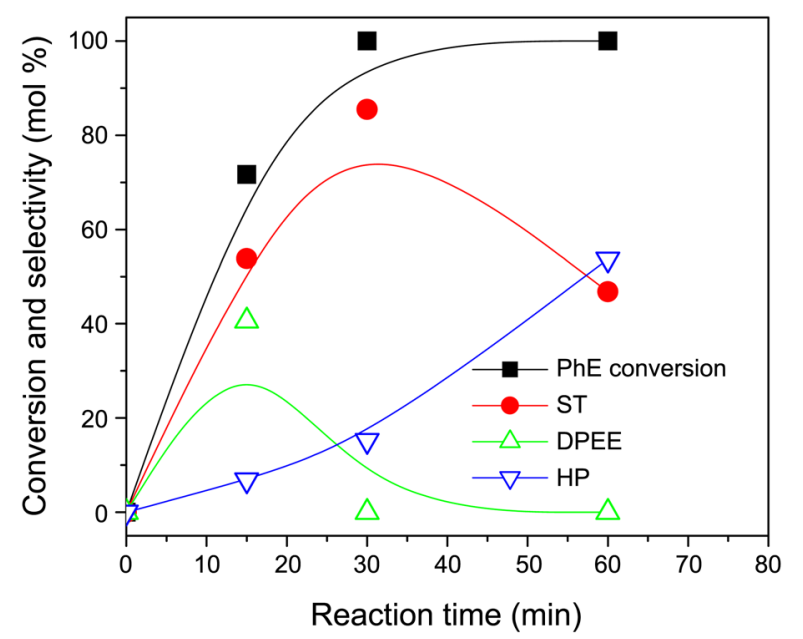

Figure 5. The $\mathrm{PhE}$ conversion and selectivities of products with time in the presence of AMB-15 catalyst when o-DCB was added as a solvent.

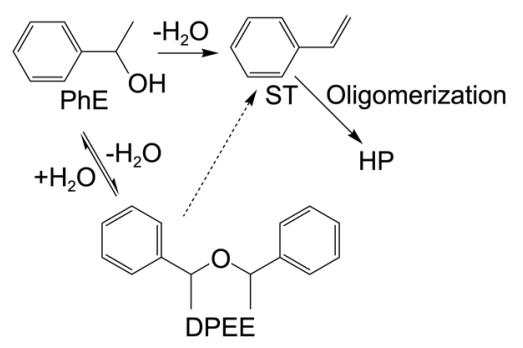

Scheme 1

Figure 4 shows that the kinetic constants for dehydration decrease in the order of $k_{\mathrm{AMB}-15}>k_{\mathrm{H}-\mathrm{Y}}>k_{\mathrm{H}-\mathrm{ZSM}-5}$, confirming the rapidest dehydration over the AMB-15 catalyst. It has been reported that the dehydration is carried out over an acid site of a catalyst. ${ }^{1,13-17}$ AMB-15 catalyst has very high acid concentration (4.7 mequiv. $\mathrm{H}^{+} / \mathrm{g}$ ) compared with the zeolites like H-ZSM-5 and H-Y. It has also been reported that the yield of ST increases with decreasing the $\mathrm{Si} / \mathrm{Al}$ ratio (or increasing acid concentration) of the H-ZSM-5 catalyst ${ }^{16}$ even though high aluminum content of H-mordenite ${ }^{14}$ zeolite is detrimental to the ST production because of difficult diffusion. Even though surface area is low $\left(45 \mathrm{~m}^{2} / \mathrm{g}\right.$, Table 1), AMB-15 is a macroporous material; ${ }^{18-22}$ therefore, AMB-15 has the advantage of rapid diffusion of reactants and products. The pore size distributions of the three catalysts shown in Supporting Figure 2 also confirm the large pore size of the AMB-15. Therefore, it can be presumed that a catalyst having high acid-site concentration and macroporosity such as acidic resins (AMB-15 and AMB-35 etc.) can be successfully used in the dehydration of PhE.

\section{Conclusions}

Following conclusions can be derived from the comparative study of the dehydration of $\mathrm{PhE}$ over the three catalysts. Resin catalyst such as AMB-15 can be successfully applied in the dehydration of PhE to produce ST because of high concentration of acid sites and ready diffusion of reactants and products. The ST selectivity can be increased further when $\mathrm{PhE}$ is diluted with a solvent because the oligomerization of ST may be hindered in low concentration. The reaction scheme for the dehydration may be summarized as follows;

- ST and DPEE are primary products and HP is a secondary product.

- DPEE, either through PhE or not, may be converted into ST.

- HP is obtained mainly from ST oligomerization.

Acknowledgments. This study was supported by a grant (B551179-10-03-00) from the cooperative R\&D Program funded by the Korea Research Council Industrial Science and Technology, Republic of Korea.

\section{References}

1. Molnár, Á.; Bartók, M. Dehydration of Alcohols, In Heterogeneous Catalysis; Sheldon, R. A., van Bekkum, H., Eds.; Wiley-VCH: Weinheim, 2001; pp 295-307.

2. Lee, K. Y.; Chae, H.-J.; Jeong, S.-Y.; Seo, G. Appl. Catal. A: General 2009, 369, 60-66.

3. Chen, D.; Moljord, K.; Fuglerud, T.; Holmen, A. Micropor. Mesopor. Mater. 1999, 29, 191-203.

4. Lee, Y.-J.; Baek, S.-C.; Jun, K.-W. Appl. Catal. A: General 2007 , 329, 130-136.

5. Jun, K.-W.; Lee, H.-S.; Roh, H.-S.; Park, S.-E. Bull. Kor. Chem. Soc. 2002, 23, 803-806.

6. Jiang, S.; Hwang, Y. K.; Jhung, S. H.; Chang, J.-S.; Hwang, J.-S.; Cai, T.; Park, S.-E. Chem. Lett. 2004, 33, 1048-1049.

7. Delsarte, S.; Grange, P. Appl. Catal. A: General 2004, 259, 269279.

8. Macias, O.; Largo, J.; Pesquera, C.; Blanco, C.; Gonzalez, F. Appl. Catal. A: General 2006, 314, 23-31.

9. Medina, E.; Bringué, R.; Tejero, J.; Iborra, M.; Fité, C. Appl. Catal. A: General 2010, 374, 41-47.

10. Chai, S.-H.; Wang, H.-P.; Liang, Y.; Xu, B.-Q. Green Chem. 2007, 9, 1130-1136.

11. Chang, J.-S.; Hong, D.-Y.; Vislovskiy, V. P.; Park, S.-E. Catal. Survey Asia 2007, 11, 59-69.

12. Kochloefl, K.; Muhler, M. Dehydrogenation of ethylbenzene, In Handbook of Heterogeneous Catalysis; Ertl, G., Knözinger, H., Schüth, F., Weitkamp, J., Eds.; 2nd ed.; Wiley-VCH: Weinheim, 2008; 7, 3229-3240.

13. Bertero, N. M.; Apesteguía, C. R.; Marchi, A. J. Catal. Commun. 2009, 10, 1339-1344.

14. Bertero, N. M.; Apesteguía, C. R.; Marchi, A. J. Catal. Commun. 2008, 10, 261-265.

15. Lange, J.-P.; Otten, V. Ind. Eng. Chem. Res. 2007, 46, 6899-6903.

16. Lange, J.-P.; Otten, V. J. Catal. 2006, 238, 6-12.

17. Romanova, R. G.; Lamberov, A. A.; Shmelev, I. G. Kinetics Catal. 2004, 45, 422-428.

18. Sharma, M. M. Reactive Funct. Polymer 1995, 26, 3-23.

19. Yoon, J. W.; Chang, J.-S.; Lee, H.-D.; Kim, T.-J.; Jhung, S. H. J. Mol. Catal. A 2006, 260, 181-186.

20. Yoon, J. W.; Jhung, S. H.; Chang, J.-S. Bull. Kor. Chem. Soc. 2008, 29, 339-341.

21. Yoon, J. W.; Jhung, S. H.; Lee, J. S.; Kim, T.-J.; Lee, H.-D.; Chang. J.-S. Bull. Kor. Chem. Soc. 2008, 29, 57-60.

22. Yoon, J. W.; Jhung, S. H.; Kim, T.-J.; Lee, H.-D.; Jang, N. H.; Chang, J.-S. Bull. Kor. Chem. Soc. 2007, 28, 2075-2078. 